\title{
Projective covers over local rings
}

\author{
Sofia Ercolanoni ${ }^{1} \cdot$ Alberto Facchini $^{1}$ (D)
}

Received: 23 October 2020 / Accepted: 22 February 2021 / Published online: 19 March 2021

(c) The Author(s) 2021

\begin{abstract}
We describe the structure of the projective cover of a module $M_{R}$ over a local ring $R$ and its relation with minimal sets of generators of $M_{R}$. The behaviour of local right perfect rings is completely different from the behaviour of local rings that are not right perfect.
\end{abstract}

Keywords Projective cover $\cdot$ Local ring $\cdot$ Minimal set of generators

Mathematics Subject Classification Primary 16D40 $\cdot$ 16E99

\section{Introduction}

There are relations in algebra between the notions of: (1) projective cover; (2) superfluous submodule; (3) left vanishing indexed family of right ideals; (4) Jacobson radical of the endomorphism ring; (5) minimal set of generators; and (6) right $T$-nilpotence. The aim of this paper is to investigate these relations when the base ring $R$ is local.

We describe the structure of projective covers of modules over a local ring (Theorem 3.1). Projective covers of a module $M_{R}$ over a local ring, when they exist, exactly correspond to minimal sets of generators (Theorem 4.1). As proved by Chwe and Neggers [5][Theorem 2 and its proof, top two paragraphs of p. 376], there is a real dichotomy in the behaviour of local rings that are right perfect and those that are not right perfect, i.e. in dealing with these notions there is a number of characterizations of right perfect rings among local rings (Theorem 4.3). For instance, a local ring is right

The second author was partially supported by Ministero dell'Istruzione, dell'Università e della Ricerca (Progetto di ricerca di rilevante interesse nazionale "Categories, Algebras: Ring-Theoretical and Homological Approaches (CARTHA)"), Fondazione Cariverona (Research project "Reducing complexity in algebra, logic, combinatorics - REDCOM" within the framework of the programme Ricerca Scientifica di Eccellenza 2018), and the Department of Mathematics "Tullio Levi-Civita" of the University of Padua (Research programme DOR1828909 "Anelli e categorie di moduli”).

Alberto Facchini

facchini@math.unipd.it

Sofia Ercolanoni

sofierco@gmail.com

1 Dipartimento di Matematica “Tullio Levi-Civita, Università di Padova, 35121 Padova, Italy 
perfect if and only if every right $R$-module has a minimal set of generators, if and only if any right $R$-module $M_{R}$ has a set of generators of cardinality exactly $\operatorname{dim}_{R / P}(M / M P)$.

The history of the main topics of this paper, that is, projective covers and minimal sets of generators, is classical and goes back to the 1960s and the 1970s. Rings over which every right module has a projective cover were characterized in the classical paper of Bass [3]; they are known as right perfect rings.

The story starts with a "dual" question: Can any linearly independent set of elements of a free module be extended to a basis? In 1970-1971, Chwe and Neggers [4, 5 ] proved that every linearly independent set of elements in a free right $R$-module can be extended to a basis by adjoining elements of a given basis if and only if the ring $R$ is local and right perfect. At about the same time, Lenzing [18] obtained the same result, and additionally showed that the seemingly weaker property that every linearly independent set of elements in a free module can be extended to a basis (by adjoining arbitrary elements) holds for precisely the same class of rings $R$. Twenty years later, Nashier and Nichols [20] characterized the same class of rings by the property that any generating set of a free right module contains a basis. Assuming that $R$ is local, it was also shown in [5] that $R$ is right perfect if and only if any right $R$-module has a minimal generating set. In fact, the argument in [5] was proving more, namely that any generating set of a right module over a right perfect local ring contains a minimal generating set; this was noticed in [20]. Another 1991 paper of Nashier and Nichols [19] provided an example of a nonlocal, nonperfect commutative ring over which every module has a minimal generating set. In the same paper [19], the authors proved that if any set of generators of a right $R$-module contains a minimal set of generators, then $R$ is perfect, and asked whether the converse implication holds.

Much more recently, abelian groups admitting a minimal generating set have been characterized by Hrbek and Růžička [13, 21], and this characterization has been extended to modules over Dedekind domains in the same authors' paper [12]. A partial characterization of modules over Dedekind domains in which every generating set has a minimal generating subset was obtained by Hrbek and Růžička in [14]. In the same paper, it was also shown that if any generating set of a free right $R$-module contains a minimal generating set, then $R$ is right perfect. In fact, the study of abelian groups in which every generating set has a minimal generating subset goes back to the 1961 paper of Dlab [6], who proved that this property characterizes primary groups of bounded order among all primary groups. The question whether torsion abelian groups of bounded order have this property remains unanswered to this day. It is a particular case of the above-mentioned question of Nashier and Nichols, which likewise remains unanswered. The latter question reduces to the following [14]: Given a module $M$ over a classically semisimple ring (like a finite matrix ring over a division ring or a finite product of division rings), does every generating set of $M$ contain a minimal generating set? This innocuously looking question, which turned out to be surprisingly difficult, is discussed by Herden, Hrbek and Růžička in [10].

Our paper was inspired from the article [8], written by Pavel Prríhoda, Zahra Nazemian and the second author, and is heavily based on the three articles $[12,14]$ and [21] by Michal Hrbek and Pavel Růžička.

In our paper, all rings are associative rings with identity and modules are unital right modules. If $R$ is a ring and $X$ is a set, the symbol $R_{R}^{(X)}$ denotes the free right $R$-module on the set $X$. 


\section{Notations and basic notions}

\subsection{Superfluous submodules of free modules.}

Let $R$ be a local ring with identity and $X$ be a set. First of all, we will consider the endomorphism ring of the free right $R$-module $F_{R}=R_{R}^{(X)}=\bigoplus_{x \in X} x R$. The endomorphism ring $E:=\operatorname{End}\left(F_{R}\right)$ of the module $F_{R}$ is isomorphic to the ring of all column-finite $X \times X$ matrices with entries in $R$. For any such matrix $A$, let $\mathcal{A}(A, x)$ denote the right ideal of $R$ generated by all entries on the $x$-th row of $A$. An indexed family of right ideals $\mathcal{I}_{x}, x \in X$, of $R$ is said to be left vanishing if for every sequence $x_{1}, x_{2}, x_{3}, \ldots$ of distinct elements of $X$ and every sequence $a_{i}$ of elements of $\mathcal{I}_{x_{i}}$ there exists a positive integer $m$ such that $a_{m} a_{m-1} \cdots a_{2} a_{1}=0$ [22]. Notice that if $X$ is finite, all families of right ideals indexed in $X$ are left vanishing.

The Jacobson radical of the endomorphism ring $E$ of the module $F_{R}$ was described in [22] by Sexauer and Warnock. They proved that the Jacobson radical $J(E)$ of the $\operatorname{ring} E$ of column-finite matrices with entries in $R$ consists of all column-finite $X \times X$ matrices $A$ with entries in $J(R)$ and for which the indexed family of right ideals $\mathcal{A}(A, x), x \in X$, is a left vanishing family of proper right ideals of $R$.

Remark 2.1 Let $\pi_{x}: R_{R}^{(X)} \rightarrow R_{R}$ denote the canonical projection of $F_{R}$ onto the $x$-th direct summand of $R_{R}^{(X)}$. For any column-finite matrix $A \in E$, let $\varphi_{A}$ indicate the endomorphism of $F_{R}$ corresponding to the matrix $A$. Then $\mathcal{A}(A, x)=\pi_{x}\left(\varphi_{A}\left(F_{R}\right)\right)$.

The remark allows us to state Sexauer and Warnock's result for local rings as follows:

Theorem 2.2 (Sexauer and Warnock [22]) Let $R$ be a local ring, $X$ be a set, and $F_{R}$ the free right $R$-module $R_{R}^{(X)}=\bigoplus_{x \in X} x R$. Then an endomorphism $\varphi$ of $F_{R}$ belongs to the Jacobson radical $J\left(\operatorname{End}\left(F_{R}\right)\right)$ if and only if the family of right ideals $\pi_{x}\left(\varphi\left(F_{R}\right)\right), x \in X$, is a left vanishing family of proper right ideals of $R$.

\section{We thus obtain a characterization of superfluous submodules of $F_{R}$ :}

Proposition 2.3 A submodule of the free right module $F_{R}=R_{R}^{(X)}=\bigoplus_{x \in X} x R$ over a local ring $R$ is a superfluous submodule of $F_{R}$ if and only if it is contained in a submodule of $F_{R}$ of the form $\bigoplus_{x \in X} x \mathcal{I}_{x}$ for some left vanishing indexed family $\left\{\mathcal{I}_{x} \mid x \in X\right\}$ of proper right ideals of $R$.

Proof $(\Rightarrow)$ Let $S$ be a superfluous submodule of $F_{R}$. Let $\pi_{x}: R_{R}^{(X)} \rightarrow R_{R}$ denote the canonical projection of $F_{R}$ onto the $x$-th direct summand of $R_{R}^{(X)}$. Then $\mathcal{I}_{x}:=\pi_{x}(S)$ is a superfluous submodule of $R_{R}$, that is, it is a proper right ideal of $R$. Clearly, $S \subseteq \bigoplus_{x \in X} x \mathcal{I}_{x}$. It remains to show that the indexed family $\left\{\mathcal{I}_{x} \mid x \in X\right\}$ is left vanishing. Suppose the contrary, i.e. that there exist a sequence $x_{1}, x_{2}, x_{3}, \ldots$ of distinct elements of $X$ and a sequence $a_{i}$ of elements of $\mathcal{I}_{x_{i}}$ such that $a_{m} a_{m-1} \cdots a_{2} a_{1} \neq 0$ for every positive integer $m$. For every $n \geq 1$ there exists $s_{n} \in S$ such that $\pi_{x_{n}}\left(s_{n}\right)=a_{n}$. The mapping $\varphi: X \rightarrow F_{R}$, defined by

$$
\varphi(x)= \begin{cases}s_{n} & \text { if } x=x_{n} \text { for some } n \geq 1, \\ 0 & \text { if } x \in X \backslash\left\{x_{n} \mid n \geq 1\right\}\end{cases}
$$


can be extended to an endomorphism $\varphi$ of $F_{R}$, and $\varphi\left(F_{R}\right) \subseteq S$. Therefore $\varphi \in J\left(\operatorname{End}\left(F_{R}\right)\right)$ [1, Proposition 17.11]. Let $A$ be the column-finite matrix associated with $\varphi$. Then $a_{n} \in \mathcal{A}\left(A, x_{n}\right)$, so that the indexed family of right ideals $\mathcal{A}(A, x), x \in X$, is not left vanishing, which contradicts Theorem 2.2.

$(\Leftarrow)$ It suffices to prove that if $\left\{\mathcal{I}_{x} \mid x \in X\right\}$ is a left vanishing indexed family of proper right ideals of $R$, then $\bigoplus_{x \in X} x \mathcal{I}_{x}$ is a superfluous submodule of $R_{R}^{(X)}$. To this end, it is sufficient to show that the canonical projection $\pi: R_{R}^{(X)} \rightarrow \bigoplus_{x \in X} x R / x \mathcal{I}_{x}$ is a projective cover. Moreover, for this, we must only prove that if $\varphi$ is an endomorphism of $F_{R}$ and $\pi \varphi=\pi$, then $\varphi$ is an automorphism of $F_{R}$ [23, Theorem 1.2.12]. Now $\pi \varphi=\pi$ implies that $\pi(\varphi-1)=0$, so that the image of the endomorphism $\varphi-1$ of $F_{R}$ is contained in $\operatorname{ker} \pi=\bigoplus_{x \in X} x \mathcal{I}_{x}$. By the result by Sexauer and Warnock [22], it follows that $\varphi-1 \in J\left(\operatorname{End}\left(F_{R}\right)\right)$. Thus $\varphi$ is an automorphism of $F_{R}$. This concludes the proof.

\subsection{Minimal sets of generators}

In this subsection, dedicated to minimal sets of generators of modules, we have chosen to be extremely elementary, to stress some easy concepts that maybe are not so clear to everybody, for instance that maximal free (=linearly independent) subsets of a module can have different cardinalities even for finitely generated modules over a commutative local artinian ring.

Consider the following three conditions on a subset $B$ of a right module $M_{R}$ over a ring $R$ :

(1) $B$ is a free set of generators for $M_{R}$.

(2) $B$ is a maximal free subset of $M_{R}$, that is, the submodule $\langle B\rangle$ of $M_{R}$ generated by $B$ is a free module with free set of generators $B$, but for every $x \in M_{R} \backslash B$ the set $B \cup\{x\}$ is linearly dependent (i.e. either $\operatorname{rann}_{R}(x) \neq 0$, or $x R \cap\langle B\rangle \neq 0$ ).

(3) $B$ is a minimal set of generators for $M_{R}$, that is, $B$ is a set of generators for $M_{R}$ and, if $b \in B$, then $B \backslash\{b\}$ generates a proper submodule of $M_{R}$.

It is well known that if $R$ is a division ring, then, for every $B \subseteq M_{R}$, the properties (1), (2) and (3) are equivalent, and they are equivalent to $B$ being a basis for the right vector space $M_{R}$. Moreover, for $R$ a division ring, bases of a vector space $M_{R}$ always exist and are equipotent, and every set of generators contains a minimal set of generators.

As is well known, the situation is completely different for a module $M_{R}$ over an arbitrary ring $R$. Modules $M_{R}$ with a subset $B$ satisfying (1) are called free modules. All right $R$-modules are free if and only if $R$ is a division ring. If $M_{R}$ is a free right module over an arbitrary ring $R$ and $B$ is a subset of $M_{R}$ satisfying (1), then $B$ also satisfies (2) and (3). It is not true that all free sets of generators of $M_{R}$ are equipotent. (Rings with this property are called IBN rings. For instance, for every infinite dimensional vector space $V_{k}$ over a division ring $k, \operatorname{End}\left(V_{k}\right)$ is not an IBN ring. Every commutative ring is an IBN ring.)

Let us pass to consider subsets $B$ of a module satisfying (2). They always exist:

Proposition 2.4 Let $M_{R}$ be any right module over an arbitrary ring $R$. Then there exists a subset $B$ of $M_{R}$ that is a maximal free subset of $M_{R}$, that is, $B$ is linearly independent, but for every $x \in M_{R} \backslash B$ the set $B \cup\{x\}$ is linearly dependent. 
Proof Notice that a subset $B$ of $M_{R}$ is free, that is, is linearly independent, if and only if $B$ is a free set of generators of the $R$-module $\langle B\rangle$, the submodule of $M_{R}$ generated by $B$. Equivalently, if the unique right $R$-module morphism $\varphi: R_{R}^{(B)} \rightarrow M_{R}$ that extends the inclusion $B \hookrightarrow M_{R}$ is an injective morphism. Let $\mathcal{F}$ be the set of all free subsets of $M_{R}$. The set $\mathcal{F}$ is non-empty, because $\emptyset \in \mathcal{F}$. Partially order $\mathcal{F}$ by set inclusion. We must show that $\mathcal{F}$ has maximal elements, and we will prove this using Zorn's lemma. Therefore it suffices to show that if $\mathcal{C} \subseteq \mathcal{F}$ is a chain in $\mathcal{F}$, that is, a subset of $\mathcal{F}$ linearly ordered by set inclusion, then $\bigcup \mathcal{C} \in \mathcal{F}$. Suppose $x_{1}, \ldots, x_{n}$ are distinct elements of $\bigcup \mathcal{C}, r_{1}, \ldots, r_{n} \in R$ and $\sum_{i} x_{i} r_{i}=0$. Since $\mathcal{C}$ is linearly ordered by set inclusion, there exists $C \in \mathcal{C}$ with $x_{1}, \ldots, x_{n} \in C$. Then $C$ is linearly independent, so that $r_{1}=\cdots=r_{n}=0$, as desired.

For modules, condition (2) does not imply (1) and condition (2) does not imply (3). For example, the only independent subset of the $\mathbb{Z}$-module $\mathbb{Z} / 2 \mathbb{Z}$ is the empty subset. Therefore $B:=\emptyset$ satisfies (2), but does not satisfy (1) and does not satisfy (3).

Subsets of $M_{R}$ satisfying (2) are not necessarily equipotent. As an example, take a suitable finitely generated free module over a ring that is not IBN. For an example over a commutative local artinian ring $R$, fix a field $k$ and the ring $R:=k[x, y] /(x, y)^{2}$. Then the $R$-module $R^{3} /((\bar{x}, 0, \bar{x}),(\bar{y}, \bar{x}, 0))$ has a maximal free set consisting of the singleton $\{(1,0,0)\}$ and a free subset $\{(0,1,0),(0,0,1)\}[17]$.

One of the aims of this paper is to study modules satisfying (3). Again we find that (3) does not imply (1) and does not imply (2). For instance, let $R:=\mathbb{Z}$ be the ring of integers and $M_{\mathbb{Z}}:=\mathbb{Z} / 6 \mathbb{Z}$, the cyclic group of order 6 . Consider the two subsets $B:=\{\overline{1}\}$ and $B^{\prime}:=\{\overline{2}, \overline{3}\}$ of $M_{R}$. Then $B$ and $B^{\prime}$ are both minimal sets of generators of $M_{\mathbb{Z}}$; hence they satisfy (3), but have different cardinalities 1 and 2. Moreover, $B$ and $B^{\prime}$ are sets of generators that are not free sets of generators, therefore they do not satisfy (1) and (2).

Thus, we have that among conditions (1), (2) and (3), only the implications (1) $\Rightarrow(2)$ and $(1) \Rightarrow(3)$ hold, and no other implication holds.

\subsection{Right $T$-nilpotence.}

The notion of left vanishing family of right ideals is clearly related to right $T$-nilpotence and its generalizations. Recall that a subset $I$ of a ring $R$ is right T-nilpotent if for each sequence $a_{1}, a_{2}, \ldots$ of elements of $I$ there exists an index $t$ such that $a_{t} \ldots a_{1}=0$. A family of right $R$-modules $\left\{M_{x} \mid x \in X\right\}$ is said to be locally semi-T-nilpotent if, for every sequence $x_{1}, x_{f_{2}}, x_{3}, \ldots$ of distinct elements of $X$, every sequence of non-isomorphisms $M_{x_{1}} \stackrel{f_{1}}{\longrightarrow} M_{x_{2}} \stackrel{f_{2}}{\longrightarrow} M_{x_{3}} \stackrel{f_{3}}{\longrightarrow} \cdots$, and every element $m \in M_{x_{1}}$, there exists an index $t \geq 1$ such that $f_{t} f_{t-1} \ldots f_{1}(m)=0$ [2]. If the same condition is also satisfied when we allow repetitions in the sequence of indices $x_{1}, x_{2}, x_{3}, \ldots$, then the family $\left\{M_{x} \mid x \in X\right\}$ is said to be locally T-nilpotent.

Recall that if $J$ is a right ideal of a ring $R$, then $J$ is right $T$-nilpotent if and only if $M J \neq M$ for every nonzero right $R$-module $M$, if and only if $\operatorname{ann}_{L}(J) \neq 0$ for every nonzero left $R$-module ${ }_{R} L$, if and only if $M J$ is superfluous in $M$ for every right $R$-module $M$, if and only if $F J$ is superfluous in $F$ for the countably generated free right $R$-module $F=R_{R}^{\left(\aleph_{0}\right)}$ ( [1, Lemma 28.3], [16, Theorem 23.16]). 


\section{Main result}

Theorem 3.1 The following conditions are equivalent for a right module $M_{R}$ over a local $\operatorname{ring} R$ :

(1) The module $M_{R}$ has a projective cover.

(2) The module $M_{R}$ is isomorphic to $F_{R} / S$, where $F_{R}=R_{R}^{(X)}=\bigoplus_{x \in X} x R$ is a free R-module, $\left\{I_{x} \mid x \in X\right\}$ is a left vanishing indexed family of proper right ideals of $R$, and $S$ is a submodule of $\bigoplus_{x \in X} x I_{x}$.

(3) The module $M_{R}$ has a minimal set of generators $X$ and there exists a left vanishing indexed family $\left\{I_{x} \mid x \in X\right\}$ of proper right ideals of $R$ such that the canonical epimorphism $\varphi: R_{R}^{(X)} \rightarrow M_{R}$ induces an isomorphism $\bigoplus_{x \in X} x R_{R} / x I_{x} \rightarrow M_{R} / \sum_{x \in X} x I_{x}$.

(4) The module $M_{R}$ has a minimal set of generators $X$ and the indexed family of right ideals $\left\{\left(\left(\sum_{x \in X \backslash\{y\}} x R\right):_{R} y\right) \mid y \in X\right\}$ is left vanishing. Here, for every $y \in X$, $\left(\left(\sum_{x \in X \backslash\{y\}} x R\right):_{R} y\right):=\left\{r \in R \mid y r \in \sum_{x \in X \backslash\{y\}} x R\right\}$.

Proof (1) $\Rightarrow$ (2) If $M_{R}$ has a projective cover $\pi: P_{R} \rightarrow M_{R}$, then $P_{R}$ is free because $R$ is local [15]. Hence $M_{R} \cong R_{R}^{(X)} / S$ for some set $X$ and some superfluous submodule $S$ of $R_{R}^{(X)}$. Proposition 2.3 allows us to conclude.

(2) $\Rightarrow(3)$ If (2) holds, there is an epimorphism $\varphi: R_{R}^{(X)} \rightarrow M_{R}$ with superfluous kernel $S$ (Proposition 2.3), so that $\varphi$ is a projective cover. Let us show that the elements $\varphi(x) \in M_{R}, x \in X$, which clearly form a set of generators for $M_{R}$, form a minimal set of generators. Fix $x_{0} \in X$. We must show that $M_{R} \neq \sum_{x \in X \backslash\left\{x_{0}\right\}} \varphi(x) R$ or, equivalently, that $R_{R}^{(X)} \neq R_{R}^{\left(X \backslash\left\{x_{0}\right\}\right)}+S$. This follows from the fact that $S$ is superfluous in $R_{R}^{(X)}$.

Now the epimorphism $\varphi$ induces an isomorphism $R_{R}^{(X)} / S \rightarrow M_{R}$, which maps the submodule $\left(\bigoplus_{x \in X} x I_{x}\right) / S$ of $R_{R}^{(X)} / S$ onto the submodule $\sum_{x \in X} x I_{x}$ of $M_{R}$. Hence $R_{R}^{(X)} /\left(\bigoplus_{x \in X} x I_{x}\right) \cong \bigoplus_{x \in X} x R_{R} / x I_{x}$ is isomorphic to $M_{R} / \sum_{x \in X} x I_{x}$.

(3) $\Rightarrow$ (4) It is enough to show that if (3) holds, then $\left(\left(\sum_{x \in X \backslash\{y\}} x R\right): y\right) \subseteq I_{y}$ for every $y \in X$. Hence, suppose $y r \in \sum_{x \in X \backslash\{y\}} x R$. Then $y r=\sum_{i=1}^{n} x_{i} r_{i}$, where $y, x_{1}, \ldots x_{n}$ are distinct elements of $X$ and $r_{1}, \ldots, r_{n}$ are in $R$. Thus the element $y r-\sum_{i=1}^{n} x_{i} r_{i}$ of $F_{R}$ is in the kernel of the canonical epimorphism $\varphi$. A fortiori, the element $y\left(r+I_{y}\right)-\sum_{i=1}^{n} x_{i}\left(r_{i}+I_{x_{i}}\right)$ of $\bigoplus_{x \in X} x R_{R} / x I_{x}$ is sent to zero by the induced morphism $\bigoplus_{x \in X} x R_{R} / x I_{x} \rightarrow M_{R} / \sum_{x \in X} x I_{x}$, which is an isomorphism, so $y\left(r+I_{y}\right)-\sum_{i=1}^{n} x_{i}\left(r_{i}+I_{x_{i}}\right)$ is the zero element of $\bigoplus_{x \in X} x R_{R} / x I_{x}$. In particular, $r \in I_{y}$, as desired.

(4) $\Rightarrow$ (1) Suppose that (4) holds. We will show that the mapping $\varphi: R_{R}^{(X)} \rightarrow M_{R}$, $\varphi: x \mapsto x$, is a projective cover. Now $\varphi$ is onto because $X$ generates $M_{R}$. Hence it suffices to show that $\operatorname{ker} \varphi$ is superfluous in $F_{R}$. By Proposition 2.3, it is enough to prove that $\operatorname{ker} \varphi \subseteq \bigoplus_{y \in X} y\left(\left(\sum_{x \in X \backslash\{y\}} x R\right): y\right)$ and that each $\left(\left(\sum_{x \in X \backslash\{y\}} x R\right): y\right)$ is a proper right ideal of $R$. It is a proper right ideal because $X$ is a minimal set of generators. Finally, if $\left(y r_{y}\right)_{y \in X}$ is an element of $F_{R}$ that is in the kernel of $\varphi$, where the elements $r_{y}$ of $R$ are almost all zero, then $\sum_{y \in X} y r_{y}$ is the zero element of $M_{R}$, and clearly $r_{y} \in\left(\left(\sum_{x \in X \backslash\{y\}} x R\right): y\right)$. This concludes the proof.

Notice that Theorem 3.1 is trivial when the right module $M_{R}$ over the local $\operatorname{ring} R$ is finitely generated. For $M_{R}$ finitely generated, the module $M_{R}$ always has a projective cover and minimal sets of generators, and all families of right ideals in the statement of the theorem are finite, hence left vanishing. 
Remark 3.2 Theorem 3.1 implies that every module with a projective cover over a local ring has a minimal set of generators. (This fact already appears in [5].) The stronger property "For a module $M_{R}$ with a projective cover over a local ring, every set of generators of $M_{R}$ contains a minimal set of generators" does not hold in general. For instance, let $R$ be a local ring that is not right perfect, and consider the projective module $M_{R}:=R_{R}^{(\mathbb{N})}$, which trivially has a projective cover. Then $M_{R}$ has sets of generators that don't contain minimal sets of generators [11, Proposition 5.22].

Corollary 3.3 A module over a local integral domain $R$ has a projective cover if and only if it is the direct sum of a finitely generated module and a free module.

Proof $(\Leftarrow)$ Every finitely generated module has a projective cover, because local rings are semiperfect [7, Theorem 3.6]. Trivially, free modules have a projective cover. Finally, the direct sum of two modules with a projective cover has a projective cover [23, Remark 1.4.2].

$(\Rightarrow)$ Assume that $M_{R}$ has a projective cover, where $R$ is a local integral domain. By Theorem 3.1, the module $M_{R}$ is isomorphic to $F_{R} / S$, where $F_{R}=R_{R}^{(X)}=\bigoplus_{x \in X} x R$ is a free $R$-module, $\left\{I_{x} \mid x \in X\right\}$ is a left vanishing indexed family of proper right ideals of $R$, and $S$ is a submodule of $\bigoplus_{x \in X} x I_{x}$. Now over an integral domain $R$, an indexed family of right ideals $I_{x}, x \in X$, is left vanishing if and only if $I_{x}=0$ for almost all indices $x \in X$. Hence there exists a finite subset $F$ of $X$ such that $I_{x}=0$ for every $x \in X \backslash F$. Thus $S \subseteq \bigoplus_{x \in F} x I_{x}$. Then $M_{R}$ is isomorphic to the direct sum of the finitely generated module $\left(\bigoplus_{x \in F} x R\right) / S$ and the free module $\bigoplus_{x \in X \backslash F} x R$.

Remark 3.4 In [11, Theorem 5.27], Hrbek characterizes the modules $M_{R}$ over a DVR $R$ (a local Dedekind domain) for which every set of generators contains a minimal set of generators, as the $R$-modules that are a direct sum of a finitely generated free $R$-module and a bounded torsion $R$-module. This allows us to easily construct:

(1) An $R$-module $M_{R}$ such that every set of generators of $M_{R}$ contains a minimal set of generators, but $M_{R}$ does not have a projective cover. (Take $M_{R}$ any bounded torsion $R$-module that is not finitely generated.) And

(2) An $R$-module $M_{R}$ with a projective cover and with a set of generators that does not contain minimal sets of generators. (Take as $M_{R}$ any free $R$-module that is not finitely generated.)

\section{Local rings, perfect rings, almost perfect rings.}

In this paper, we have restricted our attention to modules over a local ring $R$. This hypothesis considerably simplifies the setting we are considering, because:

(1) Every local ring is IBN, so that free $R$-modules have a unique rank.

(2) It is possible to attach to every right $R$-module $M_{R}$ two cardinal numbers:

(a) gen $\left(M_{R}\right)$, the smallest of the cardinalities $|X|$ of the sets $X$ of generators of $M_{R}$. It is well known that if $M_{R}$ has a minimal set $X$ of generators and $X$ is infinite, then gen $\left(M_{R}\right)=|X|$.

(b) The dimension $\operatorname{dim}_{R / P}(M / M P)$, where $P$ is the maximal ideal of $R$. 
Clearly, for any right module over a local ring $R$, one has that $\operatorname{gen}\left(M_{R}\right) \geq \operatorname{dim}_{R / P}(M / M P)$.

In the proof of Theorem 3.1, we saw that if $R$ is a local ring and $M_{R}$ is an $R$-module with a projective cover, then we can suppose that the projective cover is of the form $\varphi_{X}: R^{(X)} \rightarrow M_{R}$, where $X$ is a suitable minimal set of generators for $M_{R}$ and $\varphi_{X}$ is the unique right $R$-module morphism that extends the inclusion $X \hookrightarrow M_{R}$. Let us prove that, in this case, any minimal set of generators yields a projective cover:

Theorem 4.1 Let $R$ be a local ring and $M_{R}$ be a right $R$-module with a projective cover. Let $Y$ be any minimal set of generators of $M_{R}$. Then the canonical epimorphism $\varphi_{Y}: R^{(Y)} \rightarrow M_{R}$ is a projective cover for $M_{R}$.

Proof Let $M_{R}$ be a right module with a projective cover over a local ring $R$. Then as we have said in the paragraph before the statement of the theorem, we can suppose that the projective cover is $\varphi_{X}: R^{(X)} \rightarrow M_{R}$, where $X$ is a suitable minimal set of generators for $M_{R}$.

First case: $M_{R}$ is a free right $R$-module. If we also suppose that the module $M_{R}$ over the local ring $R$ is free, then we can assume $M_{R}=R_{R}^{(X)}$ and that $\varphi_{X}$ is the identity $1_{M_{R}}$ of $M_{R}$. Let $Y$ be a minimal set of generators of $R_{R}^{(X)}$, so that we have the canonical epimorphism $\varphi_{Y}: R_{R}^{(Y)} \rightarrow R_{R}^{(X)}$ induced by the inclusion $Y \hookrightarrow R_{R}^{(X)}$. Then $\varphi_{Y}$ is a split epi, that is, there is a direct-sum decomposition $R_{R}^{(Y)}=A \oplus B$, where $\left.\varphi_{Y}\right|_{A}: A \rightarrow R_{R}^{(X)}$ is an isomorphism, and $B$ is the kernel of $\varphi_{Y}$. The minimality of the set of generators $Y$ corresponds to the fact that $\varphi_{Y}\left(R_{R}^{(Y \backslash\{y\})}\right) \subset R_{R}^{(X)}$ for every $y \in Y$, or, equivalently, that $R_{R}^{(Y \backslash\{y\})}+B \subset R_{R}^{(Y)}$ for every $y \in Y$.

Let $P$ be the maximal ideal of $R$. Applying to the split epi $\varphi_{Y}: R_{R}^{(Y)}=A \oplus B \rightarrow R_{R}^{(X)}$ the additive right exact functor $-\bigotimes_{R} R / P: \operatorname{Mod} R \rightarrow \operatorname{Mod} R / P$, which is naturally isomorphic to the functor $X_{R} \mapsto X_{R} / X_{R} P$, we get a split epi

$$
\overline{\varphi_{Y}}: R_{R}^{(Y)} / R_{R}^{(Y)} P=\left(A+R_{R}^{(Y)} P\right) / R_{R}^{(Y)} P \oplus\left(B+R_{R}^{(Y)} P\right) / R_{R}^{(Y)} P \rightarrow R_{R}^{(X)} / R_{R}^{(X)} P,
$$

whose kernel is $\left(B+R_{R}^{(Y)} P\right) / R_{R}^{(Y)} P$ and whose restriction to $\left(A+R_{R}^{(Y)} P\right) / R_{R}^{(Y)} P$ is an isomorphism. From $R_{R}^{(Y \backslash\{y\})}+B \subset R_{R}^{(Y)}$ for every $y \in Y$, we get that $B \subseteq P_{R}^{(Y)}$, because if $B \nsubseteq P_{R}^{(Y)}$, there exist an element $y_{0} \in Y$ and an element $b=\sum_{y \in Y} y r_{y} \in B \subseteq R_{R}^{(Y)}$ with $r_{y}=0 \quad$ for almost all $y \quad$ and $r_{y_{0}} \in R \backslash P$, so $y_{0}=b r_{Y_{0}}^{-1}+\left(y_{0}-b r_{y_{0}}^{-1}\right)=b r_{y_{0}}^{-1}-\sum_{y \in Y \backslash\left\{y_{0}\right\}} y r_{y} r_{y_{0}}^{-1} \in B+R_{R}^{\left(Y \backslash\left\{y_{0}\right\}\right)}, \quad$ hence $B+R_{R}^{\left(Y Y\left\{y_{0}\right\}\right)}=R_{R}^{(Y)}$, a contradiction. The contradiction shows that $B \subseteq P_{R}^{(Y)}$, so that the direct summand $\left(B+R_{R}^{(Y)} P\right) / R_{R}^{(Y)} P$ of $R_{R}^{(Y)} / R_{R}^{(Y)} P$ is zero. Equivalently $B / B P=0$. Since $B$ is a projective $R$-module, it follows that $B=0$ [1, Proposition 17.14]. Therefore $\varphi_{Y}: R^{(Y)} \rightarrow R_{R}^{(X)}$ is an isomorphism, hence a projective cover of $R_{R}^{(X)}$.

Second case (general case): $M_{R}$ is an arbitrary module with a projective cover. Let $\varphi_{X}: R^{(X)} \rightarrow M_{R}$ be a projective cover of $M_{R}$ and $Y$ a minimal set of generators of $M_{R}$. We can suppose that $M_{R}=R^{(X)} / S$ for some superfluous submodule $S$ of $R_{R}^{(X)}$ and that $\varphi_{X}$ is the canonical projection $\pi: R^{(X)} \rightarrow R^{(X)} / S$. Since $Y$ is a minimal set of generators of $R^{(X)} / S$, we have the canonical epimorphism $\varphi_{Y}: R_{R}^{(Y)} \rightarrow R^{(X)} / S, y \mapsto y$ for every $y \in Y$. By the projectivity of $R_{R}^{(Y)}$, there exists a morphism $\psi: R_{R}^{(Y)} \rightarrow R_{R}^{(X)}$ such that $\pi \psi=\varphi_{Y}$. From the surjectivity of $\varphi_{Y}$, we get that $\psi\left(R_{R}^{(Y)}\right)+S=R_{R}^{(X)}$. But $S$ is superfluous in $R_{R}^{(X)}$, so $\psi$ is surjective. Thus $\{\psi(y) \mid y \in Y\}$ is a set of generators for $R_{R}^{(X)}$. Let us prove that it is a minimal set of generators for $R_{R}^{(X)}$. We must show that, for every $y_{0} \in Y$, the set $\left\{\psi(y) \mid y \in Y, y \neq y_{0}\right\}$ does not generate $R_{R}^{(X)}$. To this end, it suffices to prove that, for every $y_{0} \in Y$, the set $\left\{\pi \psi(y) \mid y \in Y, y \neq y_{0}\right\}$ does not generate $\pi\left(R_{R}^{(X)}\right)$. But we know that $\left\{\varphi_{Y}(y) \mid y \in Y, y \neq y_{0}\right\}$ does not generate $R_{R}^{(X)} / S$. This proves that $\{\psi(y) \mid y \in Y\}$ is a 
minimal set of generators for $R_{R}^{(X)}$. By the first case above, $\psi: R_{R}^{(Y)} \rightarrow R_{R}^{(X)}$ is a projective cover, i.e. an isomorphism. Therefore $\varphi_{Y}=\pi \psi: R_{R}^{(Y)} \rightarrow M_{R}$ is a projective cover of $M_{R}$, as desired.

Remark 4.2 Our discussion has lead us to consider, for a fixed local ring $R$ with maximal ideal $P$, four classes of right $R$-modules:

(a) The class $\mathcal{A}$ of $R$-modules with a projective cover.

(b) The class $\mathcal{B}$ of $R$-modules for which every set of generators contains a minimal set of generators.

(c) The class $\mathcal{C}$ of $R$-modules with a minimal set of generators.

(d) The class $\mathcal{D}$ of $R$-modules $M_{R}$ for which gen $\left(M_{R}\right)=\operatorname{dim}_{R / P}(M / M P)$. Let us prove that $\mathcal{A} \cup \mathcal{B} \subseteq \mathcal{C} \subseteq \mathcal{D}$.

Proof The inclusion $\mathcal{A} \subseteq \mathcal{C}$ follows from Theorem 3.1, and the inclusion $\mathcal{B} \subseteq \mathcal{C}$ is trivial. The inclusion $\mathcal{C} \subseteq \mathcal{D}$ follows from [12, Corollary 3.3] and from (2) in the first paragraph of this Section.

In our setting, there is a real dichotomy between the behaviour of perfect rings and that of nonperfect rings, as the next Theorem 4.3 shows. Most of it already appears in [5], but we prefer to give here an extremely elementary self-contained proof for completeness. See Remark 4.4.

Theorem 4.3 The following conditions are equivalent for a local ring $R$ with maximal ideal $P$ :

(1) The ring $R$ is right perfect.

(2) For every subset $X$ of any right $R$-module $M_{R}, X$ generates $M_{R}$ if and only if $\{x+M P \mid x \in X\}$ generates $M / M P$.

(3) For every right $R$-module $M_{R}$, every set of generators of $M_{R}$ contains a minimal set of generators of $M_{R}$.

(4) Every right $R$-module has a minimal set of generators.

(5) Every right $R$-module has a minimal set of generators $Y$, and for any such set $Y$ the canonical mapping $\varphi_{Y}: R_{R}^{(Y)} \rightarrow M_{R}$ is a projective cover.

(6) For every right $R$-module $M_{R}$ with a projective cover and every subset $Y$ of $M_{R}$ such that the indexed set $\{y+M P \mid y \in Y\}$ is a basis of the right vector space M/MP over the division ring $R / P$, the canonical mapping $\varphi_{Y}: R^{(Y)} \rightarrow M_{R}$ is a projective cover for $M_{R}$.

(7) $\operatorname{gen}\left(M_{R}\right)=\operatorname{dim}_{R / P}(M / M P)$ for every right $R$-module $M_{R}$.

Proof (1) $\Leftrightarrow(2)$. Condition (2) can be restated as "for the submodule $\langle X\rangle$ of $M_{R}$ generated by $X,\langle X\rangle=M_{R}$ if and only if $\langle X\rangle+M_{R} P=M_{R}$ ", which is equivalent to " $M_{R} P$ is a superfluous submodule in any module $M_{R}$ ". This is equivalent to the condition " $P$ is right $T$-nilpotent" [1, Lemma 28.3], that is, to (1).

$(2) \Rightarrow(3)$. If (2) holds, then (3) is true, because (3) is true for any vector space.

(3) $\Rightarrow(4)$ is trivial.

(4) $\Rightarrow$ (7) follows from the inclusion $\mathcal{C} \subseteq \mathcal{D}$ in Remark 4.2. 
(7) $\Rightarrow(1)$. Suppose that (1) does not hold, so that there exists a nonzero right $R$-module $M_{R}$ without maximal submodules [1, Theorem 28.4, (a) $\Leftrightarrow$ (c)]. Then $M_{R} P=M_{R}$, whence gen $\left(M_{R}\right) \neq 0$ and $\operatorname{dim}_{R / P}(M / M P)=0$. Therefore (7) does not hold.

$(1) \Rightarrow(5)$. If $R$ is right perfect, then every module has minimal sets of generators $Y$, and for any such set $Y$ the canonical mapping $\varphi_{Y}: R_{R}^{(Y)} \rightarrow M_{R}$ is a projective cover (Theorem 4.1.)

(5) $\Rightarrow(1)$. Suppose that (5) holds, and apply (5) to the right $R$-module $M_{R}:=(R / P)^{\left(\aleph_{0}\right)}$. Then the canonical basis of the $R / P$-vector space $M_{R}$ is a minimal set $X$ of generators and, by (5), the canonical mapping $\varphi: R^{\left(\aleph_{0}\right)} \rightarrow(R / P)^{\left(\aleph_{0}\right)}$ is a projective cover. Thus $P^{\left(\aleph_{0}\right)}$ is superfluous in $R^{\left(\aleph_{0}\right)}$, so $\left\{P_{n} \mid n \geq 1\right\}$, where $P_{n}=P$ for every $n$, is a left vanishing family, i.e. $P$ is right $T$-nilpotent. Hence $R$ is right perfect.

$(1) \Rightarrow(6)$. Suppose that (1) holds, so that (2) and (5) also hold. Let $Y$ be a subset of module $M_{R}$ such that the indexed set $\{y+M P \mid y \in Y\}$ is a basis of the right vector space $M / M P$ over the division ring $R / P$. By (2), $Y$ is a minimal set of generators of $M_{R}$, so that applying (5) we get (6).

(6) $\Rightarrow(1)$. Suppose that (1) does not hold, so that $R$ is not right perfect. Let $X$ be an infinite set and consider the projective right $R$-module $M_{R}:=R_{R}^{(X)}$. The identity is a projective cover $\varphi_{X}: R^{(X)} \rightarrow R^{(X)}$. The module $R^{(X)}$ has the property that not all its sets of generators contain a minimal set of generators [14, Lemma 2.2]. Let $T$ be a set of generators of $R^{(X)}$ that does not contain minimal sets of generators. The image $\bar{T}$ of $T$ via the mapping $\pi: R_{R}^{(X)} \rightarrow R_{R}^{(X)} / R_{R}^{(X)} P$ generates the vector space $R_{R}^{(X)} / R_{R}^{(X)} P$, hence contains a basis of $R_{R}^{(X)} / R_{R}^{(X)} P$. Thus there is a subset $Y$ of $T$ whose image $\bar{Y}$ via the mapping $\pi$ is a basis of $R_{R}^{(X)} / R_{R}^{R(X)} P$. But $Y$ is not a minimal set of generators for $R_{R}^{(X)}$, so that a fortiori the mapping $\varphi_{Y}: R^{(Y)} \rightarrow R^{(X)}$ is not a projective cover, and (6) does not hold for the module $M_{R}$.

Remark 4.4 Different proofs of $(1) \Rightarrow(3),(3) \Rightarrow(1)$ and $(1) \Leftrightarrow(4)$ in the previous theorem already appear in [19, 20] and [5], respectively. For instance, in [5, Theorem 2 and its proof, top two paragraphs of p. 376], Chwe and Neggers prove the following. Assume that a right $R$-module $M$ has a minimal set of generators. Then, as explained in [5, second paragraph on p. 376], one has $M P \neq M$ if $M \neq 0$. As this holds for every $R$-module $M$, one can conclude that the ideal $P$ is right $T$-nilpotent [5, Theorem $1,(1) \Leftrightarrow(4)]$. Conversely, assume that $P$ is right $T$-nilpotent; so $L P \neq P$ for any nonzero right $R$-module $L$. Let $M$ be a right $R$-module. Following [5, first paragraph on p. 376], choose a basis of the vector space $M / M P$ over $R / P$ and lift it to a subset $X$ of $M$. Let $N \subseteq M$ be the submodule generated by $X$; then $M=N+M P$, so $(M / N) P=M / N$, hence $N=M$. Any proper subset of $X$ wouldn't even generate $M / M P$; so $X$ is a minimal generating set of $M$ whose cardinality equals $\operatorname{dim}_{R / P}(M / M P)$. Moreover, the assertion that every set of generators of a module $M$ has cardinality $\geq \operatorname{dim}_{R / P}(M / M P)$ clearly holds for any module over any local ring $R$. Finally, if $M \neq 0$ has a set of generators of cardinality $\operatorname{dim}_{R / P}(M / M P)$, then $M \neq M P$; if this holds for every $M$, then $P$ is right $T$-nilpotent.

In the notation of Remark 4.2, for a local right perfect ring $R$, Theorem 4.3 asserts that $\mathcal{A}=\mathcal{B}=\mathcal{C}=\mathcal{D}=\operatorname{Mod} R$. The following proposition shows that if the ring $R$ is not right perfect, then the situation is completely different.

Proposition 4.5 In the notation of Remark4.2, for any local, not right perfect ring $R$ one has that $\mathcal{A} \nsubseteq \mathcal{B}, \mathcal{B} \nsubseteq \mathcal{A}, \mathcal{A} \cup \mathcal{B} \subset \mathcal{C}$ and $\mathcal{D} \subset \operatorname{Mod} R$. 
Proof Assume $R$ local with maximal ideal $P$ and $R$ not right perfect. To show that $\mathcal{A} \nsubseteq \mathcal{B}$ consider the free right $R$-module $R_{R}^{(X)}$ for an infinite set $X$. Then $R_{R}^{(X)}$ trivially belongs to $\mathcal{A}$ because it is projective, and $R_{R}^{(X)} \notin \mathcal{B}$ by [14, Lemma 2.2].

To prove that $\mathcal{B} \nsubseteq \mathcal{A}$ consider the right $R$-module $M_{R}:=(R / P)^{(X)}$ for some infinite set $X$. Then $M_{R} \notin \mathcal{A}$ by Theorem 3.1, and $M_{R} \in \mathcal{B}$ because the sets of generators of the $R$-module $M_{R}$ are exactly the sets of generators of the vector space $M$ over the division ring $R / P$.

In order to see that $\mathcal{A} \cup \mathcal{B} \subset \mathcal{C}$, consider the module $M_{R}:=R_{R}^{(X)} \oplus(R / P)^{(X)}$ for some infinite set $X$. Clearly, $M_{R} \in \mathcal{C}$. Also, $M_{R} \notin \mathcal{B}$, because $\mathcal{B}$ is closed under direct summands [14, p. 368]. Finally, in order to prove that $M_{R} \notin \mathcal{A}$, notice that an infinite indexed family of copies of $P$ is not left vanishing because $P$ is not right $T$-nilpotent. If $M_{R}$ has a projective cover, then any minimal set of generators of $M_{R}$ leads to a projective cover (Theorem 4.1). The $R$-module $M_{R}=R_{R}^{(X)} \oplus(R / P)^{(X)}$ has a natural minimal set of generators, indexed by the disjoint union of two copies of $X$. If this particular set of generators of $M_{R}$ leads to a projective cover, then the infinite $X$-indexed family of copies of $P$ is left vanishing by Theorem 3.1, a contradiction.

Finally, $\mathcal{D} \subset \operatorname{Mod} R$ by Theorem $4.3,(7) \Rightarrow(1)$.

We are not able to prove, in general, that $\mathcal{C} \subset \mathcal{D}$ for every local, not right perfect ring $R$. We can prove it only under the further hypothesis that $R$ is a left chain domain.

Proposition 4.6 Let $R$ be a left chain domain that is not a right perfect ring (equivalently, a left chain domain that is not a division ring). Then, in the notation of Remark 4.2, $\mathcal{C} \subset \mathcal{D}$.

(Notice that a left chain domain is right perfect if and only if it is a division ring. In fact, if $R$ is a left chain domain that is not a division ring, then there is in $P$ a nonzero element $p$. Then $p^{n} \neq 0$ for every $n$ implies that $P$ is not $T$-nilpotent.)

Proof Let $R$ be a left chain domain that is not a division ring. Then its maximal ideal $P$ contains a nonzero element $p$. Consider the direct system

$$
R_{R} \stackrel{\lambda_{p}}{\longrightarrow} R_{R} \stackrel{\lambda_{p}}{\longrightarrow} R_{R} \stackrel{\lambda_{p}}{\longrightarrow} \ldots,
$$

where $\lambda_{p}: R_{R} \rightarrow R_{R}$ is left multiplication by $p$, and let $M_{R}:=\lim R_{R}$ be the direct limit. Equivalently, $M_{R}$ is the homomorphic image of a free right $R$-module with a countable free set of generators $x_{t}, t \geq 1$, modulo the submodule generated by all the elements $x_{t}-x_{t+1} p, t \geq 1$ (Bass module). Let $\overline{x_{t}}$ denote the image of any $x_{t}$ in $M_{R}$.

Let us show that $\overline{x_{2}} \notin \overline{x_{1}} R$. We have that $\overline{x_{2}} \in \overline{x_{1}} R$ implies $\overline{x_{2}}=\overline{x_{1}} r$ for some $r \in R$, so that $\left(\lambda_{p}\right)^{n}(1)=\left(\lambda_{p}\right)^{n+1}(r)$ for some $n \geq 0$, whence $p^{n}=p^{n+1} r$. Thus we get that $p$ is right invertible, a contradiction. Hence $\overline{x_{2}} \notin \overline{x_{1}} R$.

Notice that $M_{R} P=M_{R}$, because $\overline{x_{t}}=\overline{x_{t+1}} p \in M_{R} P$ for every $t$.

Consider the module $N_{R}:=R_{R}^{\left(\aleph_{0}^{\prime}\right)} \oplus\left(M_{R} / \overline{x_{1}} R\right)$, so that $N / N P \cong(R / P)^{\left(\aleph_{0}\right)}$. Clearly, gen $\left(N_{R}\right)=\aleph_{0}$ and $\operatorname{dim}_{R / P}(N / N P)=\aleph_{0}$. Hence $N_{R} \in \mathcal{D}$. Let us show that $N_{R} \notin \mathcal{C}$. Suppose the contrary. Then $N_{R}$ has a countable minimal set of generators, $\left\{g_{n} \mid n \geq 1\right\}$ say. Every element $g_{n}$ can be written as $g_{n}=f_{n}+h_{n}$, with $f_{n} \in R_{R}^{\left(\aleph_{0}\right)}$ and $h_{n} \in M_{R} / \overline{x_{1}} R$. The set $\left\{f_{n}+N P \mid n \geq 1\right\}$ is a basis of the vector space N/NP [12, Corollary 3.3]. Now the nonzero element $\overline{x_{2}}+\overline{x_{1}} R$ of $M_{R} / \overline{x_{1}} R$ is annihilated by $p$, because $\overline{x_{2}} p=\overline{x_{1}}$. Moreover the nonzero 
element $\overline{x_{2}}+\overline{x_{1}} R$ can be written as a linear combination of the elements of the minimal set of generators:

$$
\overline{x_{2}}+\overline{x_{1}} R=\sum_{n} g_{n} r_{n}
$$

with $r_{n}=0$ for almost all $n$, but not $r_{n}=0$ for all $n$. The set of principal left ideals $\left\{R r_{n} \mid n \geq 1\right\}$ (almost all zero), has a greatest element $R r_{\bar{n}} \neq 0$. From $\operatorname{Rr}_{\bar{n}} \supseteq \operatorname{Rr} r_{n}$ for every $n \geq 1$, it follows that $r_{n}=s_{n} r_{\bar{n}}$ for all $n$, with $s_{n}=0$ for almost all $n$ and $s_{\bar{n}}=1$. Now right multiplication by $r_{\bar{n}} p$ is an endomorphism of the abelian group $N$ whose restriction to $R^{\left(\aleph_{0}\right)}$ is an injective endomorphism of $R^{\left(\aleph_{0}\right)}$ and whose restriction to $M_{R} / \overline{x_{1}} R$ is an endomorphism of $M_{R} / \overline{x_{1}} R$. Applying right multiplication by $r_{\bar{n}} p$ to the element $\sum_{n} g_{n} s_{n}$ of $N_{R}$, we get from (1) that $\left(\sum_{n} g_{n} s_{n}\right) r_{\bar{n}} p=0$. Thus $\left(\sum_{n} f_{n} s_{n}\right) r_{\bar{n}} p=0$ and $\left(\sum_{n} h_{n} s_{n}\right) r_{\bar{n}} p=0$. Hence $\sum_{n} f_{n} s_{n}=0$, so $\sum_{n}\left(f_{n}+N P\right)\left(s_{n}+P\right)=0$ in $N / N P$, where $s_{\bar{n}}+P=1_{R / P}$. This contradicts the fact that $\left\{f_{n}+N P \mid n \geq 1\right\}$ is a basis of the vector space $N / N P$.

Hrbek's characterization [11, Theorem 5.27], which we cited in Remark 3.4, is partially generalized in the next proposition. A ring $R$ is right almost perfect if $R / I$ is a right perfect ring for every proper two-sided ideal $I \neq 0$ of $R$ [9]. Every right almost perfect ring is either a prime ring or a right perfect ring. Right almost perfect rings have a number of characterizations [9, Theorem 4.6 and Proposition 4.8]. For every prime right almost perfect ring $R$, there is a natural topology on $R$ with basis of neighbourhoods of 0 all nonzero two-sided ideals of $R$. Correspondingly, there is a hereditary torsion theory on $\operatorname{Mod} R$ whose torsion modules are all semiartinian modules $A_{R}$ such that every nonzero homomorphic image of $A_{R}$ contains a simple submodule that is not faithful. The torsion-free modules are all modules $B_{R}$ whose simple submodules are all faithful. For a local prime right almost perfect ring $R$, the maximal ideal $P$ turns out to be right topologically $T$-nilpotent, in the sense that for every sequence $a_{1}, a_{2}, \ldots$ of elements of $P$ and every neighbourhood $U$ of zero there exists an index $t$ such that $a_{t} \ldots a_{1} \in U$.

Proposition 4.7 Let $R$ be a local right almost perfect ring. Let $M_{R}$ be a right $R$-module and suppose that there exists a nonzero element $r \in R$ such that $M r$ is contained in a finitely generated submodule of $M_{R}$. Then every set of generators of $M_{R}$ contains a minimal set of generators.

Proof Let $N$ be a finitely generated $R$-submodule of $M_{R}$ such that $M r \subseteq N$, where $r \in R$, $r \neq 0$. Then the right $R$-module $M / N$ is annihilated by the two-sided ideal $\operatorname{Rr} R$ of $R$, hence is a right $R / R r R$-module, and $R / R r R$ is a local right perfect ring. By [20], every set of generators of the right $R / R r R$-module $M / N$ contains a minimal set of generators. Hence the same is true for $M / N$ as a right $R$-module. Therefore it is possible to conclude by [14, Lemma 3.1].

As a corollary, if $R$ is a local right almost perfect ring and $M_{R}$ is a right $R$-module that is the direct sum of a finitely generated module and a module with a nonzero annihilator, then every set of generators of $M_{R}$ contains a minimal set of generators. This extends one of the two implications in [14, Corollary 4.7], because discrete valuation rings are local right almost perfect rings.

The authors are very grateful to Michal Hrbek for several useful suggestions. In particular, Remarks 3.2 and 3.4 are essentially due to him. 
Funding Open access funding provided by Università degli Studi di Padova within the CRUI-CARE Agreement..

Open Access This article is licensed under a Creative Commons Attribution 4.0 International License, which permits use, sharing, adaptation, distribution and reproduction in any medium or format, as long as you give appropriate credit to the original author(s) and the source, provide a link to the Creative Commons licence, and indicate if changes were made. The images or other third party material in this article are included in the article's Creative Commons licence, unless indicated otherwise in a credit line to the material. If material is not included in the article's Creative Commons licence and your intended use is not permitted by statutory regulation or exceeds the permitted use, you will need to obtain permission directly from the copyright holder. To view a copy of this licence, visit http://creativecommons.org/licenses/by/4.0/.

\section{References}

1. Anderson, D. W., Fuller, K. R.: "Rings and Categories of Modules", Second Edition, Graduate Texts in Math. 13, Springer-Verlag, New York, (1992)

2. Angeleri Hügel, L., Saorín, M.: Modules with perfect decomposition. Math. Scand. 98, 1-25 (2006)

3. Bass, H.: Finitistic dimension and a homological characterization of semi-primary rings. Trans. Amer. Math. Soc. 95, 466-488 (1960)

4. Chwe, B.S., Neggers, J.: On the extension of linearly independent subsets of free modules to bases. Proc. Amer. Math. Soc. 24, 466-470 (1970)

5. Chwe, B.S., Neggers, J.: Local rings with left vanishing radical. J. London Math. Soc. 4, 374-378 (1971)

6. Dlab, V.: On a characterization of primary abelian groups of bounded order. J. London Math. Soc. 36, 139-144 (1961)

7. Facchini, A.: Module theory. Endomorphism rings and direct sum decompositions in some classes of modules. Progress in Mathematics, vol. 167. Springer, Birkhäuser Verlag, Basel (1998). https://doi. org/10.1007/978-3-0348-8774-8

8. Facchini, A., Nazemian, Z., Prihoda, P.: Covering classes and uniserial modules, submitted for publication, 2020, available in http://arxiv.org/abs/2001.03085

9. Facchini, A., Parolin, C.: Rings whose proper factors are right perfect. Colloq. Math. 122(2), 191-202 (2011)

10. Herden, D., Hrbek, M., Ruzicka, P.: On the existence of weak bases for vector spaces. Linear Algebra Appl. 501, 98-111 (2016)

11. Hrbek, M.: Modules with a minimal generating set, diploma thesis. Univerzita Karlova v Praze, Prague (2013)

12. Weakly based modules over Dedekind domains: Hrbek, M., Růžička, P. J. Algebra 399, 251-268 (2014)

13. Hrbek, M., Ruvzivcka, P.: Characterization of Abelian groups with a minimal generating set. Quaest. Math. 38, 103-120 (2015)

14. Hrbek, M., Růžička, P.: Regularly weakly based modules over right perfect rings and Dedekind domains. Czechoslovak Math. J. 67(142), 367-377 (2017)

15. Kaplansky, I.: Projective modules. Ann. of Math 2(68), 372-377 (1958)

16. Lam, T.-Y.: A First Course in Noncommutative Rings, GTM 131. Springer-Verlag, New York (2001)

17. Lazarus, M.: Les familles libres maximales d'un module ont-elles le même cardinal? Pub. Sem. Math. Rennes 4, 1-12 (1973)

18. Lenzing, H.: A homological characterization of Steinitz rings. Proc. Amer. Math. Soc. 29, 269-271 (1971)

19. Nashier, B., Nichols, W.: A note on perfect rings. Manuscripta Math. 70, 307-310 (1991)

20. Nashier, B., Nichols, W.: On Steinitz properties. Arch. Math. (Basel) 57, 247-253 (1991)

21. Ruvzicka, P.: Abelian groups with a minimal generating set. Quaest. Math. 33(2), 147-159 (2010)

22. Sexauer, N.E., Warnock, J.E.: The radical of the row-finite matrices over an arbitrary ring. Trans. Amer. Math. Soc. 139, 287-295 (1969)

23. Xu, J.: Flat covers of modules. Lecture Notes in Math, vol. 1634. Springer-Verlag, Berlin (1996) 
Publisher's Note Springer Nature remains neutral with regard to jurisdictional claims in published maps and institutional affiliations. 